\title{
A Tunable, Simplified Model for Biological Latch Mediated Spring Actuated Systems
}

\author{
Andrés Cook, ${ }^{1}$ Kaanthi Pandhigunta, ${ }^{1}$ Mason A. Acevedo, ${ }^{1}$ Adam Walker, ${ }^{1}$ Rosalie L. Didcock, ${ }^{1}$ \\ Jackson T. Castro, ${ }^{1}$ Raghav Acharya,${ }^{2}$ M. Saad Bhamla, ${ }^{2}$ Philip S. L. Anderson, ${ }^{3}$ and Mark Ilton ${ }^{1, *}$ \\ ${ }^{1}$ Department of Physics, Harvey Mudd College, Claremont, CA 91711, USA \\ ${ }^{2}$ School of Chemical and Biomolecular Engineering, \\ Georgia Institute of Technology, Atlanta, Georgia 30318, USA \\ ${ }^{3}$ Department of Evolution, Ecology, and Behavior, \\ University of Illinois at Urbana-Champaign, Urbana, IL 61801, USA
}

(Dated: March 11, 2021)

\begin{abstract}
We develop a model of latch-mediated spring actuated (LaMSA) systems relevant to comparative biomechanics and bioinspired design. The model contains five components: two motors (muscles), a spring, a latch, and a load mass. One motor loads the spring to store elastic energy, and the second motor subsequently removes the latch, which releases the spring and causes movement of the load mass. We develop open-source software to accompany the model, which provides an extensible framework for simulating LaMSA systems. Output from the simulation includes information from the loading and release phases of motion, which can be used to calculate kinematic performance metrics that are important for biomechanical function. By rapidly iterating through biologically relevant input parameters to the model, simulated changes in kinematic performance can be used to explore the evolutionary dynamics of biological LaMSA systems and uncover design principles for bioinspired LaMSA systems.
\end{abstract}

\section{INTRODUCTION}

A diverse array of organisms use stored elastic energy to drive rapid movements. These organisms use motors, springs, and latches to perform a latch mediated spring actuated (LaMSA) motion, and remarkably, they can use this mechanism to outperform current engineering design for repeatable motion at small size-scales [1]. Models have been developed to understand the extreme biomechanics of latch-mediated spring actuated organisms. Organism-specific models, including both continuum mechanics-based models [2-10] and physical modeling with biomimetic devices [2, 10-14], have been used to test hypotheses about the mechanisms of movement in specific organisms (Table I summarizes examples of recent work).

In contrast to organism-specific models, 'simple models' with reduced complexity [15] are primarily used for making inter-species comparisons, and for testing scaling relationships and the sensitivity of kinematic performance to different characteristics of the organism. These simple models can also have broad applicability and enable the rapid testing of ideas [15], and typically include muscle motors, springs, masses, and other mechanical linkages. In recent work, these models have been applied to jumping organisms [16-23] and augmented human movements [24, 25]. General models have also been used to test hypotheses about the scaling and effectiveness of biological spring mechanisms [23, 26-29].

In our previous work [27], we used a simplified mathematical model to illustrate trade-offs between the components of a general LaMSA system. In that work, the

*milton@hmc.edu components of a LaMSA system (the latch, spring, loading motor, and load mass) were modeled as a simplified mechanical system and given material, geometric, and dynamic properties. The properties of the system components were limited to motors and springs with linear properties, specific latch shapes, frictionless interactions between components, and a fixed unlatching velocity.

Here we develop an extensible version of a simplified LaMSA system model with accompanying open-source software. Our model here includes a more general framework for defining LaMSA components (refs. [23, 26, 27, 29] are all particular cases of this new model). This broader approach allows the model to be tuned to a specific organism, group of organisms, or a biological scaling relationship to explore questions in comparative biomechanics and LaMSA system design. Our approach also includes non-linear and time-dependent material properties. Additionally, we provide a generalized treatment of the latch that includes friction, allows for different latch shapes, and includes an unlatching motor that drives the latch removal of the system, similar to the one recently hypothesized to occur in some biological systems [13].

\section{METHODS}

\section{LaMSA System Model}

In our model, the motion of a LaMSA system is comprised of three distinct phases: loading, unlatching, and spring actuation. In the loading phase (Fig. 1A, first panel), a loading motor (e.g. muscle) deforms a spring starting from the spring's stress-free equilibrium length. We make the simplifying assumption that the loading occurs slowly enough to approximate it as a quasi-static motor contraction - i.e. the loading follows the isometric 


\begin{tabular}{clr}
\hline \multicolumn{1}{c}{ Modeling Approach } & Biomechanical System & Reference \\
\hline $\begin{array}{c}\text { Continuum mechanics } \\
\text { beam bending model }\end{array}$ & click beetle latch & Bolmin et al. $(2019)[3]$ \\
\hline fluid dynamics & bladderwort trap suction feeding & Berg et al. $(2019)[4]$ \\
& Ruellia ciliatiflora seed aerodynamics & Cooper et al. $(2018)[5]$ \\
& nematocyst discharge & Hamlet et al. $(2020)[6]$ \\
\hline finite elements & locust jump & Wan and Hao $(2020)[7]$ \\
& dracula ant mandible strike & Larabee et al. $(2018)[8]$ \\
& mantis shrimp strike & Tadayon et al. $(2018)[9]$ \\
& Oxalis sp. seed ejection & Li et al. $(2020)[10]$ \\
& Oxalis sp. seed ejection & Li et al. $(2020)[10]$ \\
& bladderwort trap suction feeding & Singh et al. $(2020)[12]$ \\
& dragonfly larvae strike & Büsse et al. $(2021)[13]$ \\
& Spirostomum ambiguum contraction & Xu and Bhamla $(2019)[14]$ \\
\hline
\end{tabular}

TABLE I. Recent examples (since 2018) of modeling latch-mediated spring actuated organisms, which includes both mathematical and physical approaches. For a review of earlier work see ref. [27].

force-length curve in the case of a muscle motor. The loading phase ends when the loading motor force pushing down (in the $-y$ direction) matches the spring force pushing up. After the loading phase, the loading motor is removed from the system and the spring is held in place by a latch (Fig. 1A, second panel). The second phase of motion, the unlatching phase (Fig. 1A, third panel), begins with the activation of an unlatching motor that pulls the latch out of the way. During the unlatching phase the load mass and latch undergo a complex interaction. The interaction between the load mass and latch is modeled as a frictional contact between two rigid bodies, and the unlatching phase ends when there is no longer any contact between the load mass and latch. Once the contact breaks, the load mass is actuated solely by the spring, which undergoes a rapid expansion (Fig. 1A, fourth panel). Spring actuation continues until the spring returns to its equilibrium length where it no longer applies a force to the load mass, which causes the load mass to undergo take-off (Fig. 1A, fifth panel). In the model, we assume that the latch shape is sufficiently smooth that after the latch disengages, it does not re-engage at a later time. This assumption enables the clear delineation of the unlatching and spring actuation phases.

The dynamics of a LaMSA system depends on its components and the interaction between them. In our model, these components are classified into motors, springs, latches, and load masses (Fig. 1B). Each component is constrained to move along a single coordinate axis in the model (the loading motor, load mass and spring move along the $y$ axis; the latch and unlatching motor move along the $x$ axis). We develop our model with the aim to give general properties to each component. The motors and springs in the LaMSA system are characterized by their force output. The loading motor force $\left(F_{\mathrm{lm}}\right)$, the unlatching motor force $\left(F_{\mathrm{um}}\right)$, and spring force $\left(F_{\mathrm{sp}}\right)$ are all assumed to be functions of time, displacement, and velocity. Latches are given a shape function $y_{L}(x)$ that describes the geometry of the latch. The shape function relates horizontal motion of the latch (in the $x$ direction) to vertical displacements of the load (in the $y$ direction). Its derivatives with respect to $x$ determine the latch slope function $y_{L} \prime(x)=\frac{d y_{L}}{d x}$ and latch concavity $y_{L} \prime \prime(x)=\frac{d^{2} y_{L}}{d x^{2}}$. The functions describing shapes and forces are taken as inputs into the model to allow for hypothesis testing of non-linear properties. In addition, the mass of the system can be distributed in the spring mass $\left(m_{s}\right)$, latch mass $\left(m_{L}\right)$, and load mass $(m)$. With these definitions, we lay out the mathematical description of the model according to its three phases of motion.

\section{LaMSA Model: Loading Phase}

In the loading phase, the loading motor slowly applies a force causing a displacement of the spring. The final displacement of the spring at the end of the loading phase, $y_{0}$, is the displacement in which the loading motor force and spring force are equal and opposite, namely

$$
F_{\operatorname{lm}}\left(t=\infty, y_{0}, \dot{y}=0\right)=-F_{\mathrm{sp}}\left(t=\infty, y_{0}, \dot{y}=0\right),
$$

where $\dot{y}$ is the velocity in the $y$ direction. The condition that $t=\infty$ and $\dot{y}=0$ corresponds to a slow, quasistatic loading of the spring. The loaded displacement, $y_{0}$, depends on how the force-displacement properties of the loading motor and spring interact.

\section{LaMSA Model: Unlatching Phase}

The unlatching phase starts with the activation of the unlatching motor at time $t=0$. The load mass starts with an initial position $y=y_{0}$ and velocity $\dot{y}=0$, while the latch has an initial horizontal position $x=0$ and 


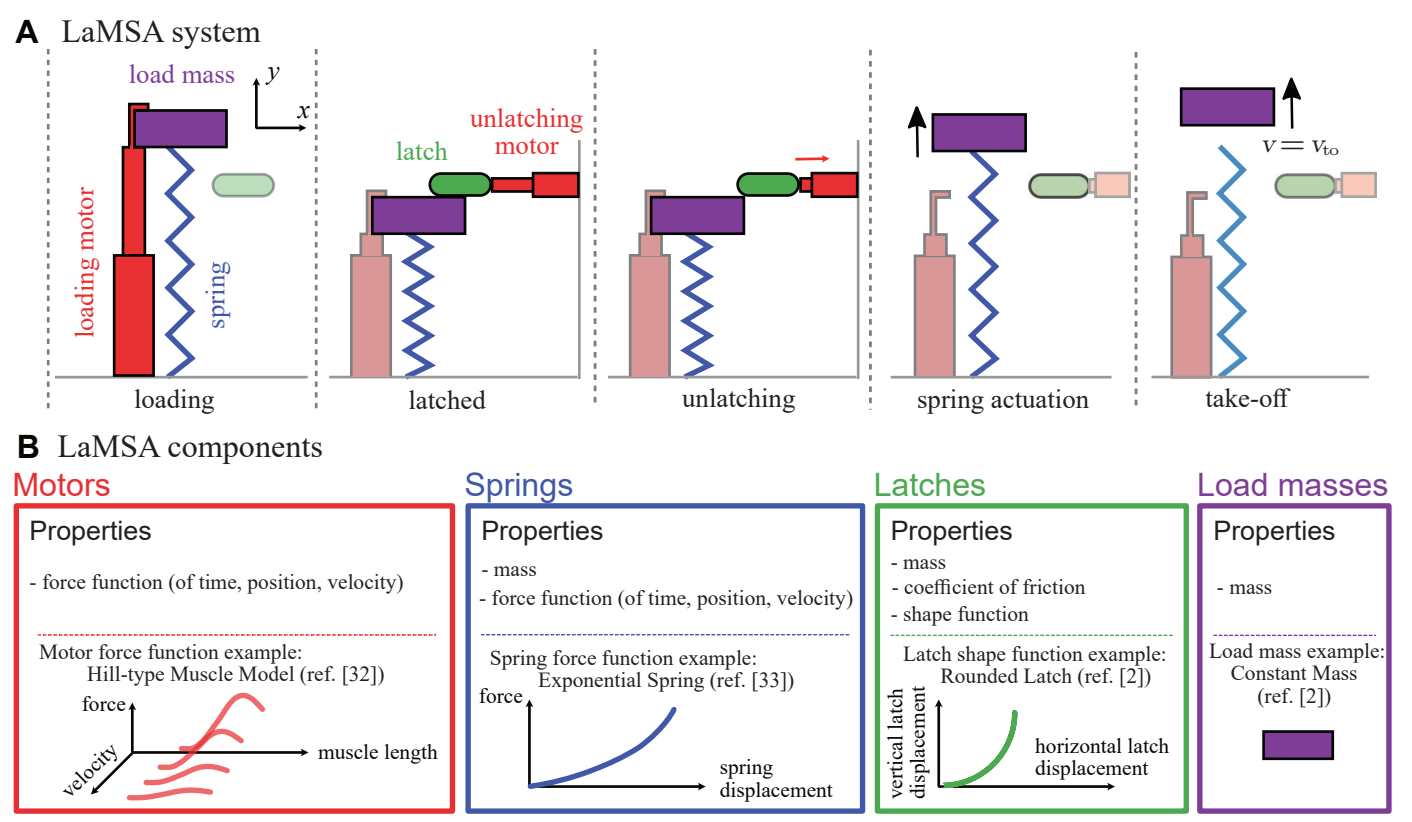

FIG. 1. Schematic description of the simplified latch-mediated spring actuated (LaMSA) system model with a loading motor, spring, latch, unlatching motor, and load mass. A The sequence of important events during the movement of a LaMSA system, which includes three delineated phases of motion in the model: loading, unlatching, and spring actuation (diagram modified from ref. [27]). B The properties of the components used in the LaMSA system model, and an example of each component that is explored in this work (see Table S1 for the specific functions and parameters used in this manuscript).

velocity $\dot{x}=v_{0}$. By analyzing the spring force pushing on the load mass, the unlatching motor force pulling on the latch, and the contact force between the load mass and latch, we derive that the differential equation for the acceleration of the latch, $\ddot{x}$, during the unlatching phase of motion

$$
\ddot{x}=\frac{\left(F_{\mathrm{um}}+F_{\mathrm{sp}} y_{L}^{\prime}-m_{\mathrm{eff}} y_{L}^{\prime} y_{L}^{\prime \prime} \dot{x}^{2}\right)+\mu_{k}\left(F_{\mathrm{um}} y_{L}^{\prime}-F_{\mathrm{sp}}+m_{\mathrm{eff}} y_{L}^{\prime \prime} \dot{x}^{2}\right)}{\left(m_{L}+m_{\mathrm{eff}}\left(y_{L}^{\prime}\right)^{2}\right)-\mu_{k}\left(m_{\mathrm{eff}} y_{L}^{\prime}-m_{L} y_{L}^{\prime}\right)} .
$$

The term $m_{\text {eff }}$ in Eq. (2) is the effective mass for the system, with $m_{\text {eff }}=m+m_{s} / 3$ [27], and $\mu_{k}$ is the coefficient of friction between the latch and load mass. A full derivation of Eq. (2) is presented in the Supplementary Materials. From the dynamics of the latch and the latch shape, the acceleration of the load mass during the unlatching phase is given by taking a chain rule, namely

$$
\ddot{y}=y_{L}^{\prime \prime} \dot{x}^{2}+y_{L}^{\prime} \ddot{x} .
$$

To determine the end of the unlatching phase, we solve for the magnitude of the normal component of the contact force between the load mass and latch,

$$
F_{N}=\frac{-m_{L} F_{\mathrm{sp}}+m_{L} m_{\mathrm{eff}} y_{L}^{\prime \prime} \dot{x}^{2}+m_{\mathrm{eff}} y_{L}^{\prime} F_{\mathrm{um}}}{m_{\mathrm{eff}} y_{L}^{\prime} \mu_{k}-m_{\mathrm{eff}}\left(y_{L}^{\prime}\right)^{2}-m_{L} \mu_{k} y_{L}^{\prime}-m_{L}} \sqrt{1+\left(y_{L}^{\prime}\right)^{2}}
$$

and require that this magnitude be $F_{N} \geq 0$ during the unlatching phase to ensure there is still contact between the load mass and latch. Therefore, we solve for when $F_{N}=0$ to determine the unlatching duration $t_{L}$, which marks the end of the unlatching phase and the beginning of the spring actuation phase of motion.

\section{LaMSA Model: Spring Actuation Phase}

After unlatching, the load mass undergoes a purely spring-driven motion given by

$$
\ddot{y}=\frac{F_{\mathrm{sp}}}{m_{\mathrm{eff}}},
$$

where the spring force can depend on position, velocity, and time. The initial conditions for this phase are given by the ending condition from the unlatching phase. Namely, for the spring actuation phase the initial position of the load mass is $y\left(t=t_{L}\right)$ and its initial velocity is $\dot{y}\left(t=t_{L}\right)$. The spring actuation phase ends when the spring stops pushing on the load mass - i.e. when $F_{\mathrm{sp}}=0$.

\section{LaMSA Model Software Implementation}

The LaMSA and direct actuation models were implemented in MATLAB. This software implementation is open-source and available at https://posmlab.github.io [30]. The software allows the user to select a LaMSA system from a library of components (motors, springs, latches, and load masses), set parameters for each component, and run a simulation to determine the dynamics of that system (as both a LaMSA system and a directly actuated system). The software can be used to iterate over the LaMSA system component parameters (e.g. spring stiffness) and rapidly generate the dynamics for variety of LaMSA systems. 

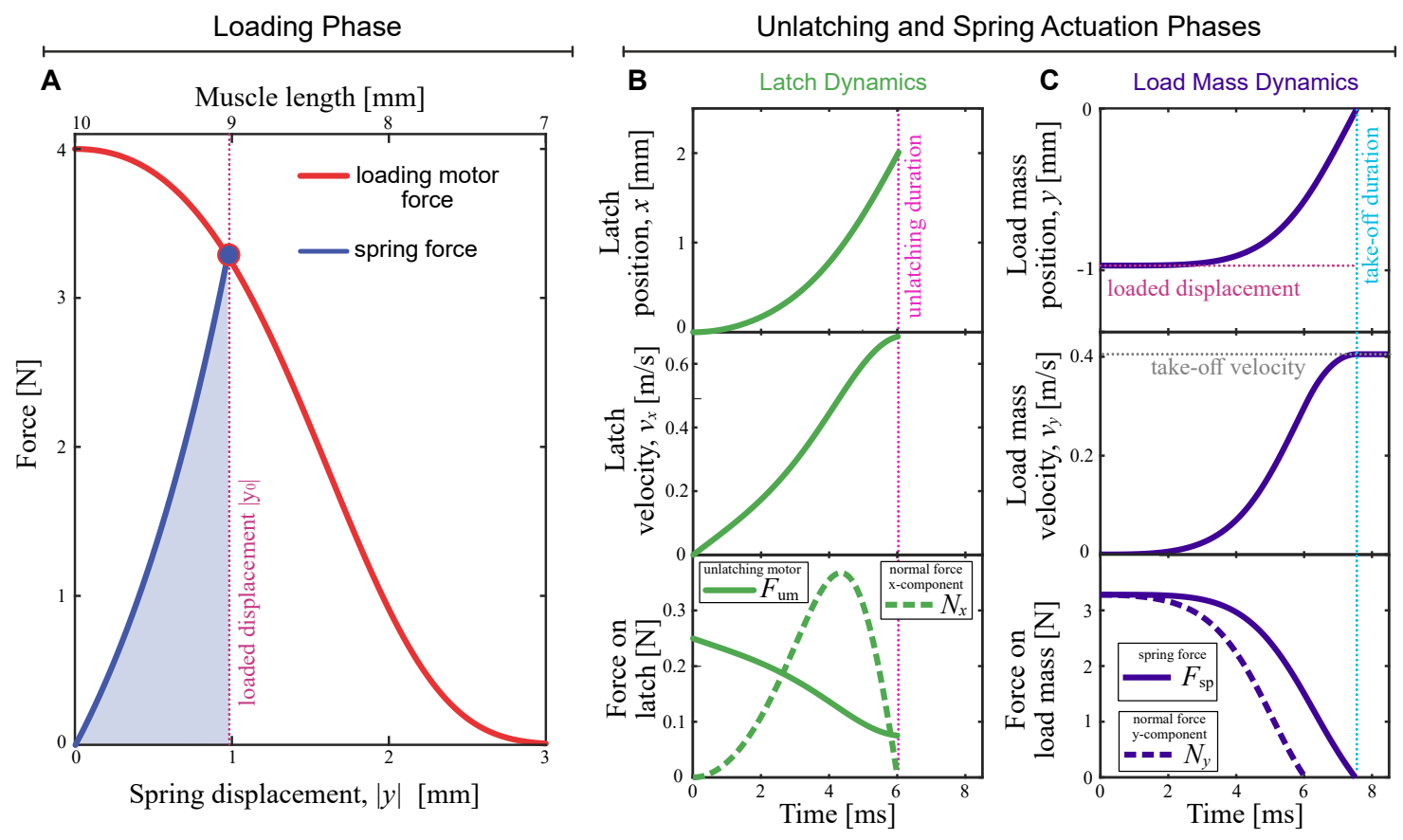

FIG. 2. Example output from the model using the components and the biological LaMSA parameters listed in Table S1. A The force-length curve for a Hill-type muscle motor loading a tendon-like exponential spring. The LaMSA system loads up to a spring displacement $y_{\max }$ calculated by equating the loading motor and spring forces. B-C The dynamics during the unlatching and spring actuation phases for the latch (panel B) and load mass (panel C). The end of the unlatching phase is marked by the pink vertical dotted line showing the unlatching duration $\left(t_{L} \approx 6 \mathrm{~ms}\right)$, which occurs when the normal force $N$ between the latch and load mass goes to zero (dashed curves in B-C). After unlatching, the load mass is actuated solely by the spring up until take-off duration $\left(t_{\text {to }} \approx 7.5 \mathrm{~ms}\right)$ when the spring force goes to zero, and the load mass reaches its take-off velocity $\left(v_{\text {to }} \approx 0.4 \mathrm{~m} / \mathrm{s}\right)$.

\section{RESULTS AND DISCUSSION}

Using the components and parameters in Table S1, the output from a single simulation generated using the software is shown in Fig. 2. The software output includes information about the loading phase, and the dynamics of the latch and load mass during the unlatching and spring actuation phases. For the load mass dynamics, the simulation generates the position $y(t)$, velocity $\dot{y}(t)$, and forces acting on the load mass. From the position and velocity of the load mass, commonly used metrics for kinematic performance in biomechanics (e.g. maximum acceleration and maximum power [1]) are calculated. The maximum load mass acceleration $(\max |\ddot{y}(t)|$, calculated from the numerical derivative of $\dot{y}(t))$ and maximum power delivered to the load mass $\left(P_{\max }=\max |m \ddot{y}(t) \dot{y}(t)|\right)$ depend on the input parameters to the model, and the open-source software enables a rapid iteration over a range of input parameters.

This modeling framework and open-source software provides an extensible platform for exploring biological LaMSA systems. Although this model was formulated generally to encompass a broad range of LaMSA systems, the model can be tuned to specific biological systems because of the flexibility in how system components are defined. The relevant range of input parameters and any interdependence between them can be informed by observed biological data and scaling. For example, depending on the system, the characteristic lengths of the system (i.e. muscle lengths, latch radius, spring length) could be constrained in the model to follow an isometric scaling. These constraints on the model can be used make inter-species comparisons and to investigate to what extent kinematic performance changes over the course of development for a given species.

Flexible component definitions also enables new components to be created that address specific biological questions. For example, Deban et al. performed a comparative analysis of tongue projection across salamander species which actuate their tongue projection with a LaMSA mechanism or by direct muscle actuation [31]. The LaMSA projection mechanism can not only lead to higher kinematic performance, but is also robust to temperature variations [31]. To explore this system with the LaMSA model presented here, new components can be created in the software that introduce a temperaturedependent motor and spring. Adding these components would yield a theoretical prediction of the relative sensitivity of the tongue projection performance to temperature for the two groups of salamanders. Comparing this 
prediction to the observed kinematics could be used to inform the modeling of how biological motors and springs depend on temperature.

Finally, the model and software presented here can offer insights into how the interrelationships between input parameters and performance may influence the evolution of these biological systems via the concept of mechanical sensitivity. Mechanical sensitivity refers to the idea that variation between parts of a multi-part system are not necessarily equal in relation to their influence on the output of the system [32,33]. Applied to a LaMSA system, we might hypothesize that variation in the spring would result in a larger variation in maximum power than variation in the latch mass. If so, that could mean that the latch mass has more freedom to evolve without altering performance. Such patterns have been identified in both mantis shrimp and fish $[33,34]$ and have been shown to influence rates of morphological evolution [35-37]. The model presented here offers an opportunity to quantitatively map how shifts in input parameters affect multiple performance metrics simultaneously, allowing for a comprehensive analysis of mechanical sensitivity.

\section{CONCLUSION}

The model and open-source software presented here balances modeling principles of simplicity and extensibility. Simplicity is provided by making explicit assumptions about how the components are connected in the model, and extensibility is achieved though flexibility of defining the individual components. With these prin- ciples, the model enables the rapid testing of ideas by simulating kinematic output across the varying model parameters. This model also opens possible new directions for future work by providing a framework for others to build upon. Case studies using the model will inform best practices for tuning the model to explore a specific biological system. Exploring biological and bioinspired LaMSA systems with this model will require input from members of comparative biomechanics community through the use of the open-software (available at https://posmlab.github.io [30]), requesting new features, and actively contributing to software development.

\section{AUTHOR CONTRIBUTIONS}

$\mathrm{AC}$ and $\mathrm{MI}$ designed the research; all authors contributed to the model development; AC, KP, MAA, AW, RLD, JTC, RA, and MI wrote the software; AC and MI wrote a first draft of the manuscript; all authors revised and edited the manuscript.

\section{ACKNOWLEDGMENTS}

This material is based upon work supported by the National Science Foundation under Grant No. 2019371. We thank the Harvey Mudd College Physics Summer Research Fund and the N. Sprague III Experiential Learning Fund for financial support. The authors thank S.N. Patek for stimulating discussions and helpful suggestions on this work.
[1] S. J. Longo, S. M. Cox, E. Azizi, M. Ilton, J. P. Olberding, R. St Pierre, and S. N. Patek, Beyond power amplification: Latch-mediated spring actuation is an emerging framework for the study of diverse elastic systems, Journal of Experimental Biology 222, 1 (2019).

[2] F. Liu, R. L. Chavez, S. N. Patek, A. Pringle, J. J. Feng, and C. H. Chen, Asymmetric drop coalescence launches fungal ballistospores with directionality, Journal of the Royal Society Interface 14, 10.1098/rsif.2017.0083 (2017).

[3] O. Bolmin, L. Wei, A. M. Hazel, A. C. Dunn, A. Wissa, and M. Alleyne, Latching of the click beetle (Coleoptera: Elateridae) thoracic hinge enabled by the morphology and mechanics of conformal structures, Journal of Experimental Biology 222, 10.1242/jeb.196683 (2019).

[4] O. Berg, K. Singh, M. R. Hall, M. J. Schwaner, and U. K. Müller, Thermodynamics of the bladderwort feeding strike - Suction power from elastic energy storage, Integrative and Comparative Biology 59, 1597 (2019).

[5] E. S. Cooper, M. A. Mosher, C. M. Cross, and D. L. Whitaker, Gyroscopic stabilization minimizes drag on Ruellia ciliatiflora seeds, Journal of the Royal Society Interface 15, 10.1098/rsif.2017.0901 (2018).
[6] C. Hamlet, W. Strychalski, and L. Miller, Fluid dynamics of ballistic strategies in nematocyst firing, Fluids 5, 1 (2020).

[7] C. Wan and Z. Hao, Natural arrangement of microstrips reduces shear strain in the locust cuticle during power amplification, Journal of Biomechanics 107, 109842 (2020).

[8] F. J. Larabee, A. A. Smith, and A. V. Suarez, Snap-jaw morphology is specialized for high-speed power amplification in the Dracula ant, Mystrium camillae, Royal Society Open Science 5, 10.1098/rsos.181447 (2018).

[9] M. Tadayon, S. Amini, Z. Wang, and A. Miserez, Biomechanical Design of the Mantis Shrimp Saddle: A Biomineralized Spring Used for Rapid Raptorial Strikes, iScience 8, 271 (2018).

[10] S. Li, Y. Zhang, and J. Liu, Seed ejection mechanism in an Oxalis species, Scientific Reports 10, 1 (2020).

[11] S. M. Cox, D. Schmidt, Y. Modarres-Sadeghi, and S. N. Patek, A physical model of the extreme mantis shrimp strike: Kinematics and cavitation of Ninjabot, Bioinspiration and Biomimetics 9, 10.1088/17483182/9/1/016014 (2014).

[12] K. Singh, R. C. Reyes, G. C. Campa, M. D. Brown, F. Hidalgo, O. Berg, and U. K. Müller, Suction flows generated 
by the carnivorous bladderwort utricularia - comparing experiments with mechanical and mathematical models, Fluids 5, 17 (2020).

[13] S. Büsse, A. Koehnsen, H. Rajabi, and S. N. Gorb, A controllable dual-catapult system inspired by the biomechanics of the dragonfly larvae's predatory strike, Science Robotics 6 (2021).

[14] L. X. Xu and M. S. Bhamla, Biophysical mechanism of ultrafast helical twisting contraction in the giant unicellular ciliate Spirostomum ambiguum, bioRxiv , 1 (2019).

[15] P. S. L. Anderson, M. D. Rivera, and A. V. Suarez, "Simple" biomechanical model for ants reveals how correlated evolution among body segments minimizes variation in center of mass as heads get larger, Integrative and Comparative Biology icaa027, 10.1093/icb/icaa027 (2020).

[16] J. P. Olberding, S. M. Deban, M. V. Rosario, and E. Azizi, Modeling the determinants of mechanical advantage during jumping: Consequences for spring- And muscledriven movement, Integrative and Comparative Biology 59, 1515 (2019).

[17] M. C. Jarur, J. Dumais, and S. Rica, Limiting speed for jumping, Comptes Rendus - Mecanique 347, 305 (2019).

[18] L. R. Davranoglou, A. Cicirello, G. K. Taylor, and B. Mortimer, Planthopper bugs use a fast, cyclic elastic recoil mechanism for effective vibrational communication at small body size, PLoS Biology 17, 1 (2019).

[19] Z. Zhang, B. Chang, J. Zhao, Q. Yang, and X. Liu, Design, Optimization, and Experiment on a Bioinspired Jumping Robot with a Six-Bar Leg Mechanism Based on Jumping Stability, Mathematical Problems in Engineering 2020, 10.1155/2020/3507203 (2020).

[20] M. Niechciał, D. Rybarczyk, and J. Buśkiewicz, Modelling the monopedal robot, IOP Conference Series: Materials Science and Engineering 710, 10.1088/1757899X/710/1/012030 (2019).

[21] C. Hong, D. Tang, Q. Quan, Z. Cao, and Z. Deng, A combined series-elastic actuator \& parallel-elastic leg nolatch bio-inspired jumping robot, Mechanism and Machine Theory 149, 103814 (2020).

[22] X. Mo, D. Romano, M. Milazzo, G. Benelli, W. Ge, and C. Stefanini, Impact of Different Developmental Instars on Locusta migratoria Jumping Performance, Applied Bionics and Biomechanics 2020, 27 (2020).

[23] G. P. Sutton, E. Mendoza, E. Azizi, S. J. Longo, J. P. Olberding, M. Ilton, and S. N. Patek, Why do large animals never actuate their jumps with latch-mediated springs? because they can jump higher without them, Integrative and Comparative Biology 59, 1609 (2019).

[24] A. Sutrisno and D. J. Braun, Enhancing Mobility with Quasi-Passive Variable Stiffness Exoskeletons, IEEE Transactions on Neural Systems and Rehabilitation Engineering 27, 487 (2019).

[25] A. Sutrisno and D. J. Braun, How to run $50 \%$ faster without external energy, Science Advances 6, 1 (2020).

[26] A. Galantis and R. C. Woledge, The theoretical limits to the power output of a muscle-tendon complex with inertial and gravitational loads, Proceedings of the Royal Society B: Biological Sciences 270, 1493 (2003).

[27] M. Ilton, M. Saad Bhamla, X. Ma, S. M. Cox, L. L. Fitchett, Y. Kim, J. sung Koh, D. Krishnamurthy, C. Y. Kuo, F. Z. Temel, A. J. Crosby, M. Prakash, G. P. Sutton, R. J. Wood, E. Azizi, S. Bergbreiter, and S. N. Patek, The principles of cascading power limits in small, fast biological and engineered systems, Science 360, 10.1126/science.aao1082 (2018).

[28] E. M. Abbott, T. Nezwek, D. Schmitt, and G. S. Sawicki, Hurry up and get out of the way! exploring the limits of muscle-based latch systems for power amplification, Integrative and Comparative Biology 59, 1546 (2019).

[29] S. Divi, X. Ma, M. Ilton, R. S. Pierre, B. Eslami, S. N. Patek, and S. Bergbreiter, Latch-based control of energy output in spring actuated systems, Journal of the Royal Society Interface 17, 20200070 (2020).

[30] R. L. Didcock, K. Pandhigunta, M. Ilton, J. T. Castro, M. A. Acevedo, A. Cook, and A. Walker, MATLAB LaMSA Model, 10.5281/zenodo.3978584 (2020).

[31] S. M. Deban, J. A. Scales, S. V. Bloom, C. M. Easterling, M. K. O'Donnell, and J. P. Olberding, Evolution of a high-performance and functionally robust musculoskeletal system in salamanders, Proceedings of the National Academy of Sciences of the United States of America 117, 10445 (2020).

[32] M. A. Koehl, When does morphology matter?, Annual Review of Ecology and Systematics 27, 501 (1996).

[33] P. S. Anderson and S. N. Patek, Mechanical sensitivity reveals evolutionary dynamics of mechanical systems, Proceedings of the Royal Society B: Biological Sciences 282, 10.1098/rspb.2014.3088 (2015).

[34] Y. Hu, N. Nelson-Maney, and P. S. Anderson, Common evolutionary trends underlie the four-bar linkage systems of sunfish and mantis shrimp, Evolution 71, 1397 (2017).

[35] M. M. Muñoz, P. S. Anderson, and S. N. Patek, Mechanical sensitivity and the dynamics of evolutionary rate shifts in biomechanical systems, Proceedings of the Royal Society B: Biological Sciences 284, 10.1098/rspb.2016.2325 (2017).

[36] M. M. Muñoz, Y. Hu, P. S. Anderson, and S. N. Patek, Strong biomechanical relationships bias the tempo and mode of morphological evolution, eLife 7, 1 (2018).

[37] M. M. Muñoz, The Evolutionary Dynamics of Mechanically Complex Systems, Integrative and Comparative Biology 59, 705 (2019).

[38] M. V. Rosario, G. P. Sutton, S. N. Patek, and G. S. Sawicki, Muscle-spring dynamics in time-limited, elastic movements, Proceedings of the Royal Society B: Biological Sciences 283, 10.1098/rspb.2016.1561 (2016).

[39] J. A. Monroy, K. L. Powers, C. M. Pace, T. Uyeno, and K. C. Nishikawa, Effects of activation on the elastic properties of intact soleus muscles with a deletion in titin, Journal of Experimental Biology 220, 828 (2017). 
SUPPLEMENTARY MATERIAL

\section{Supplementary Materials: Table of Parameters Used}

\begin{tabular}{|c|c|c|}
\hline \multicolumn{3}{|c|}{ Loading Motor } \\
\hline \multicolumn{3}{|c|}{ Force Function: (ref. [38]) } \\
\hline \multicolumn{3}{|c|}{$F_{\operatorname{lm}}(t, y, \dot{y})=F_{\max } \exp \left(-\left|\left(\left(\frac{L_{i}-y}{L_{o}}\right)^{b}-1\right) / s\right|^{a}\right)\left(\frac{1}{1}\right.$} \\
\hline \multicolumn{3}{|c|}{ Default Loading Motor Parameters Used: } \\
\hline$F_{\max }=4 \mathrm{~N}$ & $v_{\max }=1 \mathrm{~m} / \mathrm{s}$ & $L_{o}=L_{i}=10 \mathrm{~mm}$ \\
\hline$a=2.08$ & $b=-2.89 \quad s=-0.75$ & $r_{\text {act }}=200 \mathrm{~s}^{-1}$ \\
\hline
\end{tabular}

Spring
Force Function: $($ ref. $[39])$
$F_{\mathrm{sp}}(t, y, \dot{y})=\left\{\begin{array}{l}l_{c} k_{0} e^{-y / l_{c}}-1, \text { for } F_{\mathrm{sp}}<F_{\mathrm{s}, \max } \\ F_{\mathrm{s}, \max }, \text { otherwise }\end{array}\right.$
$\begin{array}{ll}\text { Default Spring } \text { Parameters Used: } \\ l_{c}=1 \mathrm{~mm} \quad k_{0}=2 \mathrm{kN} / \mathrm{m} \quad F_{\mathrm{sp}, \max }=20 \mathrm{~N} \quad m_{s}=2 \mathrm{~g}\end{array}$

\begin{tabular}{|l|l|}
\hline Latch \\
\hline Shape Function: (ref. [27]) \\
$y_{L}(x)=R\left(1-\sqrt{1-x^{2} / R^{2}}\right)$ \\
Default Latch Parameters Used: \\
$R=5 \mathrm{~mm} \quad m_{L}=3 \mathrm{~g} \quad \mu_{k}=0$ & \\
\end{tabular}

\begin{tabular}{|l|}
\hline Unlatching Motor \\
Force Function: $($ ref. $[27])$ \\
$F_{\mathrm{um}}(t, x, \dot{x})=\left\{\begin{array}{l}F_{\max }\left(1-\frac{\dot{x}}{v_{\max }}\right), \text { for } 0 \leq x \leq d \\
0, \text { otherwise }\end{array}\right.$ \\
$\begin{array}{l}\text { Default Unlatching Motor Parameters Used: } \\
F_{\max }=0.25 \mathrm{~N} \quad v_{\max }=1 \mathrm{~m} / \mathrm{s}\end{array}$ \\
\hline
\end{tabular}

\begin{tabular}{|l|}
\hline Load Mass \\
\hline Default Load Mass Parameters Used: \\
$m=10 \mathrm{~g}$ \\
\hline
\end{tabular}

TABLE S1. Mathematical description of the LaMSA components and default parameter values used in this work. The parameters were selected based on the range of characteristic forces, lengths, and velocities for biological LaMSA systems [27]. 


\section{Supplementary Materials: Derivation of the Model}

Here we consider a simplified Latch Mediated Spring Actuated (LaMSA) system:

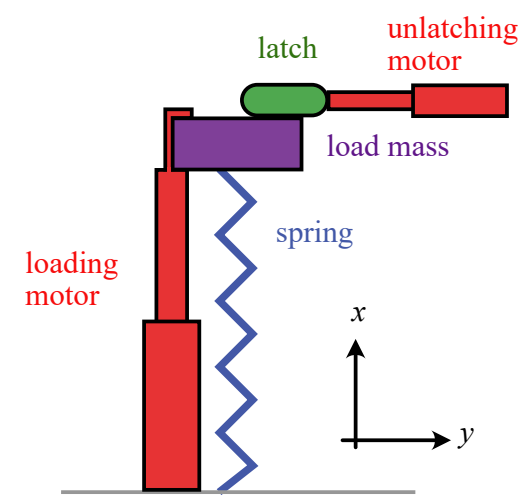

Our goal is to derive a single ordinary differential equation describing $\mathrm{x}(\mathrm{t})$ of the latch while it is in contact with the load mass.

\section{Setting Up the Problem}

Let us approximate the load mass and latch as point masses and draw isolation diagrams. In this model, we will consider the latch to have some shape that governs the unlatching process. Therefore, we have some function $y_{L}(x)$ that determines the curve of the latch.

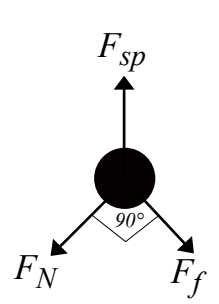

Load Mass

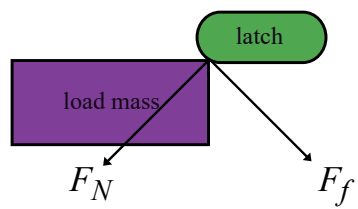

Variables:

$m_{\mathrm{eff}}=m_{\text {load }}+\frac{m_{\mathrm{spring}}}{3}:$ the effective mass of the load mass and spring mass combined $F_{\mathrm{sp}}$ : force exerted by the spring on the load mass

$\mu_{k}:$ coefficient of friction between the load mass and the latch

$F_{N}$ : normal force

$F_{f}:$ force of friction between latch and load mass

$\theta$ : The angle between the normal force vector and the vertical

Among these variables, we'll consider the following to be given:

$F_{\mathrm{sp}}, \mu_{k}$

With names for our variables, we can write Newton's second law to get the following:

$$
\begin{aligned}
\sum F_{y}= & m_{\mathrm{eff}} \ddot{y}=F_{\mathrm{sp}}-F_{N_{y}}-F_{f_{y}} \\
& m_{\mathrm{eff}} \ddot{y}=F_{\mathrm{sp}}-F_{N} \cos \theta-\mu_{k} F_{N} \cos (90-\theta) \\
& m_{\mathrm{eff}} \ddot{y}=F_{\mathrm{sp}}-F_{N} \cos \theta-\mu_{k} F_{N} \sin \theta
\end{aligned}
$$



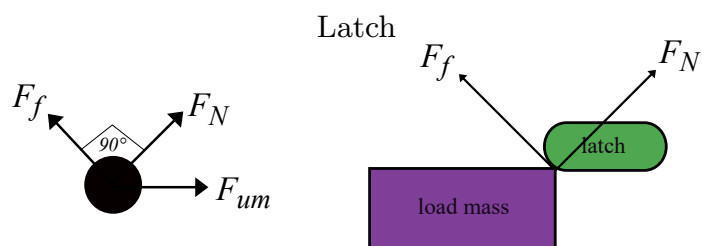

Variables:

$m_{L}$ : mass of the latch

$F_{\text {um }}$ : force of the unlatching motor pulling the latch away

$F_{N}$ : Normal force from load mass on latch

$F_{f}:$ Friction force from load mass on latch

$y_{L}(x)$ : function describing the latch geometry

Known Values:

$m_{L}:$ mass of the latch

$F_{\text {um }}$ : force of the unlatching motor pulling the latch away

$y_{L}(x)$ : function describing the latch geometry

We get the following equations from Newton's 2nd Law:

$$
\begin{aligned}
\sum F_{x}= & m_{L}(\ddot{x})=F_{\mathrm{um}}+F_{N_{x}}-F_{f_{x}} \\
m_{L}(\ddot{x}) & =F_{\mathrm{um}}+F_{N} \sin \theta-\mu_{k} F_{N} \sin (90-\theta) \\
m_{L}(\ddot{x}) & =F_{\mathrm{um}}+F_{N} \sin \theta-\mu_{k} F_{N} \cos \theta
\end{aligned}
$$

Rewriting Unknowns in Terms of Other Variables

We will use these replacements later in the derivation.

Rewriting $\ddot{y}$ - Our goal is to get a differential equation for $\ddot{x}$, but we will end up with $\ddot{y}$ in our equations. So, we can use the following to rewrite $\ddot{y}$ in terms of $\ddot{x}$ and the latch curve:

$$
\begin{aligned}
\ddot{y} & =\frac{d}{d t}\left(\frac{d y}{d t}\right) \\
& =\frac{d}{d t}\left(\frac{d y}{d x} \cdot \frac{d x}{d t}\right) \\
& =\frac{d}{d t}\left(y_{L}^{\prime} \cdot \dot{x}\right) \\
& =y_{L}^{\prime \prime} \cdot \dot{x}^{2}+y_{L}^{\prime} \cdot \ddot{x}
\end{aligned}
$$

Rewriting $\tan \theta-$ We will need to replace $\tan \theta$ later in the derivation.

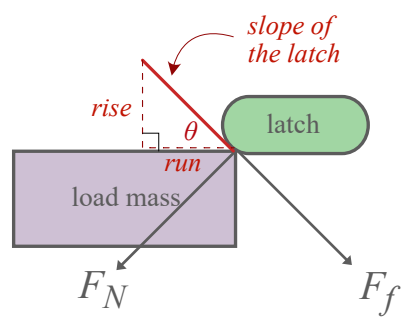

Because the latch geometry is described by the function $y_{L}$, the slope of the latch is described by the derivative $y_{L}^{\prime}$.

$$
\begin{array}{r}
y_{L}^{\prime}=\frac{\text { rise }}{\text { run }} \\
\tan \theta=\frac{\text { rise }}{\text { run }} \\
\tan \theta=y_{L}^{\prime}
\end{array}
$$




\section{Solving for $\ddot{x}$}

Solving for $F_{N}$ in each equation- Recall that we obtained the following two equations from applying Newton's 2nd Law to both :

$$
\begin{aligned}
m_{\mathrm{eff}}(\ddot{y}) & =F_{\mathrm{sp}}-F_{N} \cos \theta-\mu_{k} F_{N} \sin \theta \\
m_{L}(\ddot{x}) & =F_{\mathrm{um}}+F_{N} \sin \theta-\mu_{k} F_{N} \cos \theta
\end{aligned}
$$

Let us replace $\ddot{y}$ with the expression we obtained in the previous section. Now we have:

$$
\begin{aligned}
m_{\mathrm{eff}}\left(y_{L}^{\prime \prime} \cdot \dot{x}^{2}+y_{L}^{\prime} \cdot \ddot{x}\right) & =F_{\mathrm{sp}}-F_{N} \cos \theta-\mu_{k} F_{N} \sin \theta \\
m_{L}(\ddot{x}) & =F_{\mathrm{um}}+F_{N} \sin \theta-\mu_{k} F_{N} \cos \theta
\end{aligned}
$$

The only element that is not known is $F_{N}$. Let us eliminate it by solving for $F_{N}$ in both equations. Solving for $F_{N}$ in Eq. (6) gives us:

$$
F_{N}=\frac{F_{\mathrm{sp}}-m_{\mathrm{eff}}\left(y_{L}^{\prime \prime} \cdot \dot{x}^{2}+y_{L}^{\prime} \cdot \ddot{x}\right)}{\cos \theta+\mu_{k} \sin \theta}
$$

Solving for $F_{N}$ in Eq. (7) gives us:

$$
F_{N}=\frac{m_{L} \ddot{x}-F_{\mathrm{um}}}{\sin \theta-\mu_{k} \cos \theta}
$$

Expressing $F_{N}$ without using $\ddot{x}$ - While it is our ultimate goal to solve for $\ddot{x}$, a side goal that is useful for determining the end of the unlatching phase is obtaining an expression for $F_{N}$ that does not include $\ddot{x}$.

We can achieve this by taking Eqs. (6) and (7) from the previous section, isolating $\ddot{x}$ in each, and setting them equal to each other.

Rearranging Eq. (6):

$$
\begin{aligned}
m_{\mathrm{eff}}\left(y_{L}^{\prime \prime} \cdot \dot{x}^{2}+y_{L}^{\prime} \cdot \ddot{x}\right) & =F_{\mathrm{sp}}-F_{N} \cos \theta-\mu_{k} F_{N} \sin \theta \\
m_{\mathrm{eff}} y_{L}^{\prime} \ddot{x} & =F_{\mathrm{sp}}-F_{N} \cos \theta-\mu_{k} F_{N} \sin \theta-m_{e f f} y_{L}^{\prime \prime} \dot{x}^{2} \\
\ddot{x} & =\frac{F_{\mathrm{sp}}-F_{N} \cos \theta-\mu_{k} F_{N} \sin \theta-m_{e f f} y_{L}^{\prime \prime} \dot{x}^{2}}{m_{e f f} y_{L}^{\prime}}
\end{aligned}
$$

Rearranging Eq. (7):

$$
\begin{aligned}
m_{L}(\ddot{x}) & =F_{\mathrm{um}}+F_{N} \sin \theta-\mu_{k} F_{N} \cos \theta \\
\ddot{x} & =\frac{F_{\mathrm{um}}+F_{N} \sin \theta-\mu_{k} F_{N} \cos \theta}{m_{L}}
\end{aligned}
$$

Setting these equal to each other, isolating $F_{N}$ :

$$
\begin{aligned}
& \frac{F_{\mathrm{sp}}-F_{N} \cos \theta-\mu_{k} F_{N} \sin \theta-m_{e f f} y_{L}^{\prime \prime} \dot{x}^{2}}{m_{e f f} y_{L}^{\prime}}=\frac{F_{\mathrm{um}}+F_{N} \sin \theta-\mu_{k} F_{N} \cos \theta}{m_{L}} \\
& m_{L}\left(F_{\mathrm{sp}}-F_{N} \cos \theta-\mu_{k} F_{N} \sin \theta-m_{e f f} y_{L}^{\prime \prime} \dot{x}^{2}\right)=m_{e f f} y_{L}^{\prime}\left(F_{\mathrm{um}}+F_{N} \sin \theta-\mu_{k} F_{N} \cos \theta\right) \\
& -F_{N} m_{L} \cos \theta-F_{N} m_{L} \mu_{k} \sin \theta-F_{N} m_{e f f} y_{L}^{\prime} \sin \theta+F_{N} m_{e f f} y_{L}^{\prime} \mu_{k} \cos \theta= \\
& m_{L} m_{e f f} y_{L}^{\prime \prime} \dot{x}^{2}-m_{L} F_{\mathrm{sp}} \\
& +m_{\text {eff }} y_{L}^{\prime} F_{\mathrm{um}} \\
& F_{N}\left(m_{e f f} y_{L}^{\prime} \mu_{k} \cos \theta-m_{e f f} y_{L}^{\prime} \sin \theta-m_{L} \mu_{k} \sin \theta-m_{L} \cos \theta\right)= \\
& m_{L} m_{e f f} y_{L}^{\prime \prime} \dot{x}^{2}-m_{L} F_{\mathrm{sp}} \\
& +m_{e f f} y_{L}^{\prime} F_{\mathrm{um}} \\
& F_{N}=\frac{m_{L} m_{e f f} y_{L}^{\prime \prime} \dot{x}^{2}-m_{L} F_{\mathrm{sp}}+m_{e f f} y_{L}^{\prime} F_{\mathrm{um}}}{m_{e f f} y_{L}^{\prime} \mu_{k} \cos \theta-m_{e f f} y_{L}^{\prime} \sin \theta-m_{L} \mu_{k} \sin \theta-m_{L} \cos \theta}
\end{aligned}
$$


It's somewhat inconvenient to have $\theta$ in this expression, so we can make the following substitutions based on the geometry of our problem:

$$
\sin \theta=\frac{y_{L}^{\prime}}{\sqrt{1+\left(y_{L}^{\prime}\right)^{2}}} \quad \cos \theta=\frac{1}{\sqrt{1+\left(y_{L}^{\prime}\right)^{2}}}
$$

Plugging these in:

$$
F_{N}=\frac{m_{L} m_{e f f} y_{L}^{\prime \prime} \dot{x}^{2}-m_{L} F_{\mathrm{sp}}+m_{e f f} y_{L}^{\prime} F_{\mathrm{um}}}{m_{e f f} y_{L}^{\prime} \mu_{k}-m_{e f f}\left(y_{L}^{\prime}\right)^{2}-m_{L} \mu_{k} y_{L}^{\prime}-m_{L}} \sqrt{1+\left(y_{L}^{\prime}\right)^{2}}
$$

Great! Now that we have this, we'll resume with our other goal, of solving for $\ddot{x}$.

Solving for $\ddot{x}$ - With two expressions for $F_{N}$ in Eqs. (6) and (7), we can set them equal to each other to solve for $\ddot{x}$ :

$$
\frac{F_{\mathrm{sp}}-m_{\mathrm{eff}}\left(y_{L}^{\prime \prime} \cdot \dot{x}^{2}+y_{L}^{\prime} \cdot \ddot{x}\right)}{\cos \theta+\mu_{k} \sin \theta}=\frac{m_{L} \ddot{x}-F_{\mathrm{um}}}{\sin \theta-\mu_{k} \cos \theta}
$$

Cross-multiply to get:

Expanding:

$$
\left(\sin \theta-\mu_{k} \cos \theta\right)\left(F_{\mathrm{sp}}-m_{\mathrm{eff}}\left(y_{L}^{\prime \prime} \cdot \dot{x}^{2}+y_{L}^{\prime} \cdot \ddot{x}\right)=\left(\cos \theta+\mu_{k} \sin \theta\right)\left(m_{L} \ddot{x}-F_{\mathrm{um}}\right)\right.
$$

$$
\begin{aligned}
& F_{\mathrm{sp}} \sin \theta-m_{\mathrm{eff}} \sin \theta\left(y_{L}^{\prime \prime} \cdot \dot{x}^{2}+y_{L}^{\prime} \cdot \ddot{x}\right)-F_{\mathrm{sp}} \mu_{k} \cos \theta+m_{\mathrm{eff}} \mu_{k} \cos \theta\left(y_{L}^{\prime \prime} \cdot \dot{x}^{2}+y_{L}^{\prime} \cdot \ddot{x}\right)= \\
& m_{L} \ddot{x} \cos \theta-F_{\mathrm{um}} \cos \theta+m_{L} \mu_{k} \ddot{x} \sin \theta-F_{\mathrm{um}} \mu_{k} \sin \theta
\end{aligned}
$$

Divide both sides by $\cos \theta$ :

$$
\begin{gathered}
\left.F_{\mathrm{sp} \sin \theta-}-m_{\mathrm{efffin}} \tan \operatorname{ty}_{L}^{\tan } \cdot \dot{x}^{2}+y_{L}^{\prime} \cdot \ddot{x}\right)-F_{\mathrm{sp}} \mu_{k} \cos \theta+m_{\mathrm{eff}} \mu_{k} \cos \theta\left(y_{L}^{\prime \prime} \cdot \dot{x}^{2}+y_{L}^{\prime} \cdot \ddot{x}\right) \\
=m_{L} \ddot{x} \cos \theta-F_{\mathrm{um} \cos \theta}+m_{L} \mu_{k} \ddot{x} \sin \theta-F_{\mathrm{um}} \mu_{k} \sin \theta \\
F_{\mathrm{sp}} \tan \theta-m_{\mathrm{eff}} \tan \theta\left(y_{L}^{\prime \prime} \cdot \dot{x}^{2}+y_{L}^{\prime} \cdot \ddot{x}\right)-F_{\mathrm{sp}} \mu_{k}+m_{\mathrm{eff}} \mu_{k}\left(y_{L}^{\prime \prime} \cdot \dot{x}^{2}+y_{L}^{\prime} \cdot \ddot{x}\right) \\
=m_{L} \ddot{x}-F_{\mathrm{um}}+m_{L} \mu_{k} \ddot{x} \tan \theta-F_{\mathrm{um}} \mu_{k} \tan \theta
\end{gathered}
$$

We can replace $\tan \theta$ with $y_{L}^{\prime}$ :

$$
\begin{array}{r}
F_{\mathrm{sp}} y_{L}^{\prime}-m_{\mathrm{eff}} y_{L}^{\prime}\left(y_{L}^{\prime \prime} \cdot \dot{x}^{2}+y_{L}^{\prime} \cdot \ddot{x}\right)-F_{\mathrm{sp}} \mu_{k}+m_{\mathrm{eff}} \mu_{k}\left(y_{L}^{\prime \prime} \cdot \dot{x}^{2}+y_{L}^{\prime} \cdot \ddot{x}\right) \\
=m_{L} \ddot{x}-F_{\mathrm{um}}+m_{L} \mu_{k} \ddot{x} y_{L}^{\prime}-F_{\mathrm{um}} \mu_{k} y_{L}^{\prime}
\end{array}
$$

If we expand the equation, move all terms that contain $\ddot{x}$ and $\dot{x}^{2}$ to one side, and factor out $\ddot{x}$ and $\dot{x}^{2}$, we get:

$$
\begin{array}{r}
\ddot{x}\left(m_{\mathrm{eff}} \mu_{k} y_{L}^{\prime}-m_{\mathrm{eff}}\left(y_{L}^{\prime}\right)^{2}-m_{L}-m_{L} \mu_{k} y_{L}^{\prime}\right)+\dot{x}^{2}\left(m_{\mathrm{eff}} \mu_{k} y_{L}^{\prime \prime}-m_{\mathrm{eff}} y_{L}^{\prime} y_{L}^{\prime \prime}\right) \\
=-F_{\mathrm{um}}-F_{\mathrm{um}} \mu_{k} y_{L}^{\prime}-F_{\mathrm{sp}} y_{L}^{\prime}+F_{\mathrm{sp}} \mu_{k}
\end{array}
$$

Now, let us solve for $\ddot{x}$ and regroup some terms to arrive at the final result:

$$
\ddot{x}=\frac{\left(F_{\mathrm{um}}+F_{\mathrm{sp}} y_{L}^{\prime}\right)+\mu_{k}\left(F_{\mathrm{um}} y_{L}^{\prime}-F_{\mathrm{sp}}\right)-\dot{x}^{2}\left(m_{\mathrm{eff}} y_{L}^{\prime} y_{L}^{\prime \prime}-m_{\mathrm{eff}} \mu_{k} y_{L}^{\prime \prime}\right)}{\left(m_{L}+m_{\mathrm{eff}}\left(y_{L}^{\prime}\right)^{2}\right)-\mu_{k}\left(m_{\mathrm{eff}} y_{L}^{\prime}-m_{L} y_{L}^{\prime}\right)}
$$

And if there is no friction such that $\mu_{k}=0$ :

$$
\ddot{x}=\frac{\left(F_{\mathrm{um}}+F_{\mathrm{sp}} y_{L}^{\prime}\right)-\dot{x}^{2}\left(m_{\mathrm{eff}} y_{L}^{\prime} y_{L}^{\prime \prime}\right)}{m_{L}+m_{\mathrm{eff}}\left(y_{L}^{\prime}\right)^{2}}
$$

